\title{
A LINEAR PROGRAMMING APPROACH TO MAXIMUM ENTROPY SIGNAL RESTORATION
}

\author{
Gary A. Mastin and Dennis C. Ghiglia \\ Sandia National Laboratories \\ P. O. Box 5800 \\ Albuquerque, NM 87185
}

SAND--88-0371C

DE88 013021

\begin{abstract}
In future computing environments where computer resources are abundant, a linear programming (LP) approach to maximum entropy signal/image restoration could have advantages over traditional techniques. A revised simplex LP algorithm with inequality constraints is presented.

Dantzig's bounded-variable method is used to express the maximum entropy restoration problem as a LP problem. This is done by approximating the nonlinear objective function with piecewise linear segments, then bounding the variables as a function of the number of segments used. Linear inequality constraints may be used to assure a basic feasible solution.

Experimental results with 512-point signals are presented. These include restorations of noisy signals. Problems with as many as 513 equations and 6144 unknowns are demonstrated. The complexity of the LP restoration approach is briefly addressed.
\end{abstract}

\section{Introduction}

-...when we make inferences based on incomplete information, we should draw them from that probability distribution that has the maximum entropy allowed by the information we do have."[1]

The fundamental task in signal restoration is to replace information which is lost through a gignal degradation process. This must be done within constraints imposed by the signal and the degradation process. Only in rare cases is there exact knowledge of the missing data. We are usually resigned to making the "best possible" estimate of what should be used to restore the signal. "Best" is a qualitative judgement and there are many quantitative criteria for defining "best." One definition is based on the well known fact that natural systems tend toward energy states which maximize entropy [2]. Maximum entropy signal restoration techniques are an attempt to mimic nature by maximixing the entropy of the restored information subject to constraints imposed by the problem.

An important point must be made which can affect the layman's belief about the appropriateness of the maximum entropy technique. A maximum entropy restoration is not necessarily a maximum probability restoration. In other words, the signal restored by a maximum entropy technique is usually not the most probable restored signal. It is the restoration which can be combinatorily formed in the most number of ways [3]. If maximum probability is not the required criteria, then maximum entropy restoration is appropriate.

There is considerable controversy over the form of the entropy expression. The issue is whether $-f \ln f$ or $-\ln f$ is the proper form, $f$ representing signal amplitude. Kikuchi and Soffer [4] examine this argument in great depth and provide detailed mechanisms for determining which form is appropriate for a given problem. This analysis is well beyond the scope of this report. The convention in the image restoration field is to use the form $-f \ln f$. The majority of the image restoration problems posed as maximum entropy problems have occured in optical astronomy and the $-f \ln f$ form is supported by Kikuchi and Soffer for this application. Experience has shown that even a crude approximation to $-f \ln f$ works well, as long as the function remains convex. Results presented later in this report show that reducing the number of piecewise linear segments used to approximate the entropy function to just two segments has little effect on the restoration.

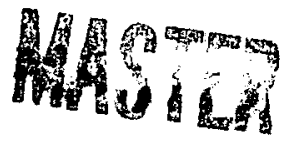




\section{DISCLAIMER}

This report was prepared as an account of work sponsored by an agency of the United States Government. Neither the United States Government nor any agency Thereof, nor any of their employees, makes any warranty, express or implied, or assumes any legal liability or responsibility for the accuracy, completeness, or usefulness of any information, apparatus, product, or process disclosed, or represents that its use would not infringe privately owned rights. Reference herein to any specific commercial product, process, or service by trade name, trademark, manufacturer, or otherwise does not necessarily constitute or imply its endorsement, recommendation, or favoring by the United States Government or any agency thereof. The views and opinions of authors expressed herein do not necessarily state or reflect those of the United States Government or any agency thereof. 


\section{DISCLAIMER}

Portions of this document may be illegible in electronic image products. Images are produced from the best available original document. 
With the question, "Why maximum entropy as a restoration criteria?", addressed and main controversies mentioned for completeness, we will now formulate the maximum entropy signal restoration problem. Once posed, the applicability of Dantzig's bounded-variable linear programming (LP) approach will be addressed. Posing a problem is only half the battle: implementing it is the next issue. We will present the LP problem in a form which may be implemented using a large revised-simplex package. The report will then be concluded with test results and analysis.

\section{Problem Formulation}

Our problem is formulated in the spirit of Frieden [5] who was the first to pose the signal restoration problem as a maximum entropy problem. A number of other formulations exist and the reader should refer to [6-10] for details. Let us begin by defining the signal values

$$
f_{j} ; 0<f_{j} \leq 1 ; j=1, \ldots, J
$$

where each $f_{j}$ is an unperturbed, noiseless input to the imaging system. The actual signal amplitude is simply rescaled between 0 and 1 here for use in the entropy relation. The input signal undergoes perturbation by a linear detection system. The point-spread-function (PSF), which is a matrix representation for the detection system degradation characteristics, is known or has been estimated. The degraded signal may then be represented by the relation

$$
\sum_{j=1}^{J} f_{j} H_{j k}+n_{j}=g_{j} ; j, k=1, \ldots, J
$$

where $H_{j k}$ is a space-invariant PSF (Toeplitz form), $n_{j}$ is a positive additive noise, and $g_{j}$ (the output of the convolution equation) is the detected, degraded signal. By adding an amplitude bias, the requirement $n_{j}>0$ does not impose any restrictions on real-world data.

Our goal is to maximise the entropy of the restored signal given constraints imposed by the convolution equation (2). We may identify two components in the objective function to be maximized: one due to the input signal and one due to noise. We assume that the signal and noise are independent. With this in mind, we may state our restoration objective as

$$
\operatorname{MAX}\left\{z=\sum_{j=1}^{J}-f_{j} \ln f_{j}+\sum_{j=1}^{J}-n_{j} \ln n_{j}\right\}
$$

or

$$
M I N\left\{z=\sum_{j=1}^{J} f_{j} \ln f_{j}+\sum_{j=1}^{J} n_{j} \ln n_{j}\right\}
$$

where the $M A X$ and $M I N$ are taken over the variables $f_{j}, n_{j}, j=1,2, \ldots, J$.

Two more constraints are needed. The first is an energy constraint to assure that there is no gain in the restored signal. The total energy, $P_{0}$, has the form

$$
\sum_{j=1}^{J} f_{j}=P_{0}
$$

In reality, we don't know the exact value of $P_{0}$. We estimate $P_{0}$ by summing the degraded signal intensities, $g_{j}$. An exact value of $P_{0}$ is not critical. The second relates to the signal-to-noise ratio (SNR) of the degraded signal relative to the input signal. Let

$$
\rho=\sum_{j=1}^{J} f_{j} / \sum_{j=1}^{J} n_{j}
$$


be our SNR estimate. Because of the positivity constraint imposed for linear programming, we know that any noise corrupted signal value, $g_{j}$, will have an amplitude greater than or equal to that of the signal value, $f_{j}$. We may set an upper bound on $f_{j}$ based on our SNR estimate, which is

$$
f_{j} \leq g_{j}-g_{j} / \rho ; j=1,2, \ldots, J
$$

The final form of the problem is, therefore,

$$
\operatorname{MIN}\left\{z=\sum_{j=1}^{J} f_{j} \ln f_{j}+\sum_{j=1}^{J} n_{j} \ln n_{j}\right\}
$$

subject to

$$
\begin{gathered}
\sum_{j=1}^{J} f_{j} H_{j k}+n_{j}=g_{j} ; j, k=1, \ldots, J \\
\sum_{j=1}^{J} f_{j}=P_{0} \\
g_{j}-g_{j} / \rho \geq f_{j} \\
f_{j}>0 ; n_{j}>0 ; j=1, \ldots, J .
\end{gathered}
$$

Most researchers pose this problem with equality constraints and solve it using Lagrange multipliers. If, however, one can approximate the objective function with piecewise linear segments, the resulting system can be solved by an LP technique. Posing the problem in this way allows one to introduce inequality constraints which can be a distinct advantage for very large problems.

\section{Implementing Dantzig's Bounded-Variable Method}

The problem posed by equations (8) with a nonlinear objective function, but linear constraints, is not new to LP applications. Kuhn and Tucker [11] and Tucker [12] showed that if the concave objective function were nonnegative and differentiable, then the method of Lagrange multipliers could be extended to inequality constraints on concave functions. Dantrig [13] considered convex functions having nonpositive values (negative of concave functions) and relaxed the requirement of differentiability provided that several conditions on the convex set are satisfied. Dantzig [13] further developed the convex objective function approach to include separable convex objectives. The bounded variable method is one such approach. It has the advantage of keeping the number of equations fixed, while increasing the number of variables with the number of separable objectives. When dealing with LP techniques, increasing tableau columns is always a computational advantage over increasing tableau rows.

We may state the LP restoration problem of equations (8) using Dantrig's bounded-variable method fairly simply. The properties of convex functions which form the theoretical basis of this method are not discussed here. The reader is refered to reference [13] for a complete discussion. We shall begin with a mathematical description of the piecewise linear approximation to a general convex function, extend the description to our maximum entropy function, then present our LP restoration problem in the form used in the experiments which are discussed in the following section.

Let $\bar{\varphi}(x)$ be a piecewise linear approximation to a general continuous convex function $\varphi(x)$. We will momentarily restrict our discussion to $x$ having only one component, $x_{j}$. This is in contrast to the real restoration problem in which $x$ is a vector having $J$ components. Let $\bar{\varphi}\left(x_{j}\right)$ have break-point coordinates $\left(f_{r j}, \bar{\varphi}\left(f_{r j}\right)\right), r=0,1, \ldots, k+1$, where $k$ is the number of piecewise linear segments (Figure 1). Any $x_{j}$ bounded by $f_{0 j}$ and $f_{r} ;$ may be represented as

$$
x_{j}=\sum_{r=0}^{k} \lambda_{r j} f_{r j}
$$


where

$$
\sum_{r=0}^{k} \lambda_{r j}=1
$$

and

$$
\lambda_{r j} \geq 0
$$

Note that for the case shown in Figure $1, \lambda_{3 j}$ through $\lambda_{k j}$ are zero and $\lambda_{2 j}=\left(1-\lambda_{1 j}\right)$.

The general LP problem, where $x$ is now a vector having $J$ components, may then be stated as

$$
\begin{gathered}
M I N\left\{z=\sum_{j=1}^{J} \overline{\varphi_{j}}\left(x_{j}\right)\right\} \\
=M I N\left\{z=\sum_{j=1}^{J} \sum_{r=0}^{k} \lambda_{r j} \bar{\varphi}\left(f_{r j}\right)\right\}
\end{gathered}
$$

where

$$
x_{j}=\sum_{r=0}^{k} \lambda_{r j} f_{r j}
$$

subject to

$$
\begin{gathered}
\sum_{j=1}^{J} a_{i j}\left[\lambda_{0 j} f_{0 j}+\lambda_{1 j} f_{1 j}+\ldots+\lambda_{k j} f_{k j}\right]=g_{j} \\
\sum_{r=0}^{k} \lambda_{r j}=1 ; j=1, \ldots, J \\
\lambda_{r j} \geq 0 .
\end{gathered}
$$

where $a_{i j}$ is the cost matrix. We solve the LP problem, then compute $x_{j}$ from equation (9) to complete the solution.

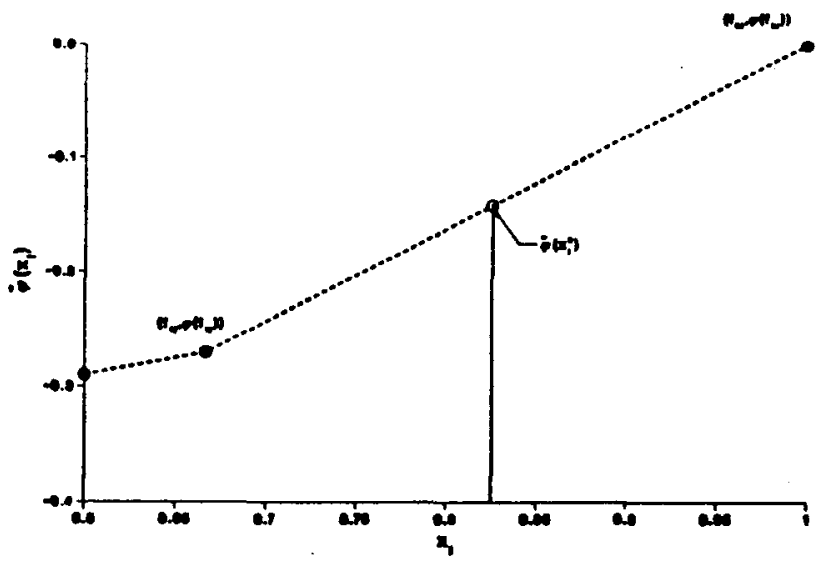

Figure 1. Arbitrary objective function having breakpoint coordinates $\left(f_{r j}, \varphi\left(f_{r j}\right)\right) . x_{j}$ is bounded by $f_{1 j}$ and $f_{2 j}$. The superscript 0 denotes simplex iteration number 0 .

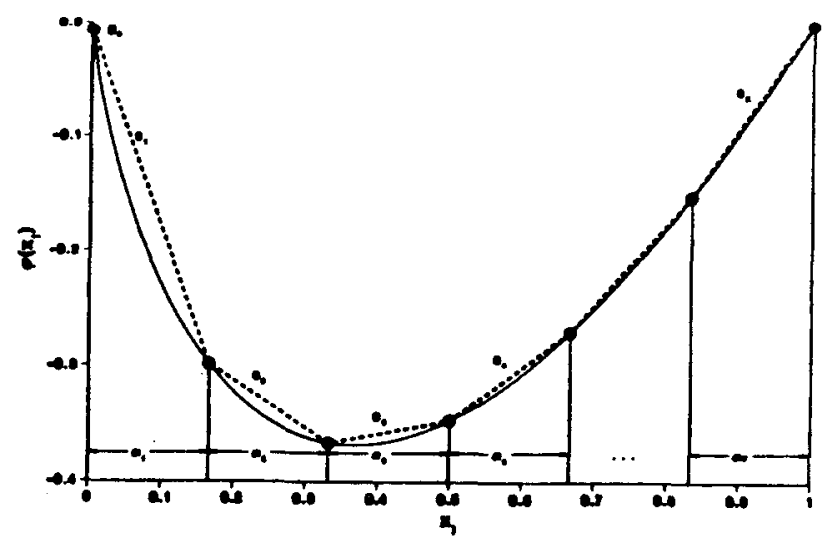

Figure 2. Convex segmented objective function and it's associated bounded variables [13]. 
The primary drawback to posing the signal restoration problem in the form of equations (12) is that we increase the number of equations by one for each $\overline{\varphi_{j}}\left(x_{j}\right)$ as well as increasing the number of variables by the number of segments. We may take advantage of the linearisation resulting from a segmented convex function while keeping the original number of equations by exploiting Dantzig's upper bounding method. Each variable in the constraint equations is replaced by a set of bounded variables, the number of bounded variables being a function of the number of convex segments. Figure 2 graphically shows a convex segmented objective function and it's associated bounded variables. Dantzig's formulation of the linear programming problem follows.

Let $s_{r}$ represent the slopes of the piecewise linear segments approximating the convex function and let $\alpha_{r}$ represent the widths (upper bounds) of the traparoids defined by the segments in Figure 2. If the segmented objective function is convex, then

$$
s_{1} \leq s_{2} \leq \ldots \leq s_{k}
$$

We can see that

$$
\bar{\varphi}\left(x_{j}\right)=g_{0}+M I N\left[s_{1} \Delta_{1}+s_{2} \Delta_{2}+\ldots+s_{k} \Delta_{k}\right]
$$

where

$$
x_{j}=\Delta_{1}+\Delta_{2}+\ldots+\Delta_{k}, \quad 0 \leq \Delta_{r} \leq \alpha_{r} .
$$

The minimum is determined by choosing $\Delta_{1}$ as large as possible $\left(\Delta_{1}=\alpha_{1}\right), \Delta_{2}$ as large as possible $\left(\Delta_{2}=\alpha_{2}\right)$, etc. until the $\Delta$ associated with the last trapasoid, $\Delta_{k}$, is chosen to be $\leq \alpha_{k}$ in order to satisfy equation (15). By picking $\Delta_{r}$ in this way, we can see that $\bar{\varphi}\left(x_{j}\right)$ is generated exactly.

The signal restoration problem posed in equations (8) may be restated in a bounded variable, segmented objective function form. Define the vector $x$ to include the unperturbed signal and the additive noise component,

$$
\vec{x}=\left[f_{1}, f_{2}, \ldots, f_{J}, n_{1}, n_{2}, \ldots, n_{J}\right]^{T}
$$

and the objective function,

$$
\begin{gathered}
M I N\left\{z=\sum_{j=1}^{J} f_{j} \ln f_{j}+\sum_{j=1}^{J} n_{j} \ln n_{j}\right\} \\
=\sum_{j=1}^{2 J} \bar{\varphi}\left(x_{j}\right) \\
=\sum_{j=1}^{2 J}\left[s_{1 j} \Delta_{1 j}+s_{2 j} \Delta_{2 j}+\ldots+s_{k j} \Delta_{k j}\right]
\end{gathered}
$$

where the first subscript is the segment number and the second subscript is the vector component number. We can restate the constraint relation, shown in equations (8), as

$$
\sum_{j=1}^{2 J} a_{i j} x_{j}=b_{i} \quad ; i=1, \ldots, J+1
$$

where $\vec{x}$ is defined by equation (16),

$$
b=\left[g_{1}, g_{2}, \ldots, g_{J}, P_{0}\right]^{T}
$$

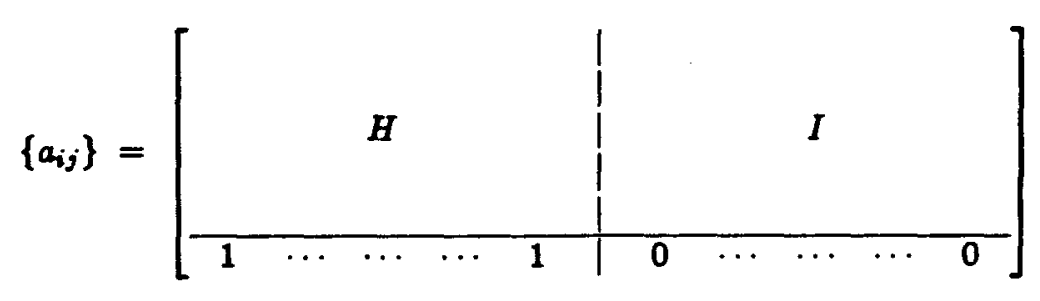


and $H$ is the Toeplitz matrix from the first constraint of equations (17) and $I$ is the identity matrix. Note also that equation (18) includes the energy constraint of equation (5).

The SNR constraint is not explicitly expressed in equations (17) and (18). It is incorporated in bounds set on the $b_{i}$ in the revised simplex LP implementation. Just as we set bounds on the variables comprising $x_{j}$, we may set bounds on $b_{i}$. In this case, we will set a lower bound

$$
L B\left(b_{i}\right)=0
$$

and an upper bound

$$
U B\left(b_{i}\right)=b_{i}-b_{i} / \rho .
$$

Because $x_{j}$ in equation (18) is formed by the sum of $k$ segmented variables, the matrix expression for the constraint equations could contain horizontal summations of $\Delta_{k j}$. This is undesirable and can be eliminated by repeating each column in $\left\{a_{i j}\right\} k$ times and by making $\vec{x}$ a column vector containing $2 J k$ entries. Adding columns, as opposed to rows, does not appreciably increase the computation time of a revised simplex LP algorithm. It does, however, significantly increase memory requirements.

Dantrig's bounded-variable method has been implemented using a revised simplex LP algorithm. The LP approach is applicable because, as shown above, the nonlinear objective function is easily decoupled into a set of piecewise linear functions. We used the LP algorithm named SPLP [14] because it offered a number of advantages over other LP algorithms. The advantages include 1) applicability to moderately large problems, 2) handling of degeneracy, 3) public domain availability, 4) machine portability, 5) relative ease of use, and 6) robustness.

\section{Experimental Results}

Restoration algorithms such as the one presented here perform differently as a result of 1) the correctness of the PSF estimate and 2) noise corruption of the signal. To evaluate the performance of the LP approach, two sets of tests were done. The first tested it's performance in the noiseless case where the restoration PSF differed from the true PSF. A rectangular PSF was used in each case, but the restoration PSF varied up to $\pm 20 \%$ from the true PSF. The second test measured the algorithm's performance in the presence of noise. The same signal was blurred by a 21-element rectangular PSF, then differing amounts of uniformly distributed noise were added. In both sets of tests, 3- and 6-segment approximations to the entropy function were used.

The PSF test showed the algorithm to be fairly intolerant of errors in the PSF estimate. The quality of these restorations differed only slightly as a function of the number of segments approximating the entropy function. When the PSF is known exactly, the test signal restorations are perfect. Figure 3 shows 3-segment restorations when the PSF is known and when the estimate is $19 \%$ wider than the true PSF.

Restoration quality analysis of blurred, noisy signals depends on the invertigator's goal. Locations of point sources can be discerned in higher noise signals than can the structures of extended sources. We were not especially impressed with restorations of our test signal (Fig. 3a) when the SNR was below 23. Common characteristics of the restored signals are attenuation of point sources, poor resolution of adjacent narrow Gaussians, and deformations of extended sources such as the sine lobe in Figure 3. Figure 4 shows a blurred, noigy signal with \& SNR of 10.99 along with the 6-segment restored signal. Note the stair-step pattern on the side of the restored sine lobe. This is a function of the number of segments approximating the entropy function.

The complexity of the LP restoration approach should not be overlooked. Restoration algorithms based on revised-simplex linear programming are much more organizationally and computationally complex than transform-based restorations. One must be constantly sensitive to a host of tolerance and scaling parameters. These may need to be adjusted for different classes of signals and can require some trial and error processing. Secondly, nonlinear restorations are complex by their very nature. Several of our test signals showed characteristics which are unexplained due to the nonlinear nature of the problem, but which are qualitatively reminiscent of "ringing" in unconstrained inverse linear filtering problems. 


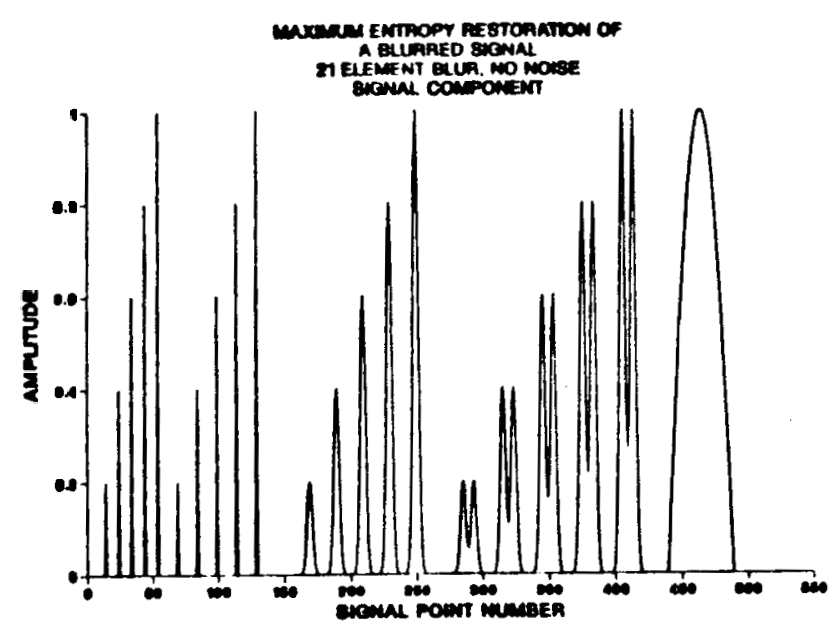

Figure 3. a) 3-8egment restoration when the true PSF is known.

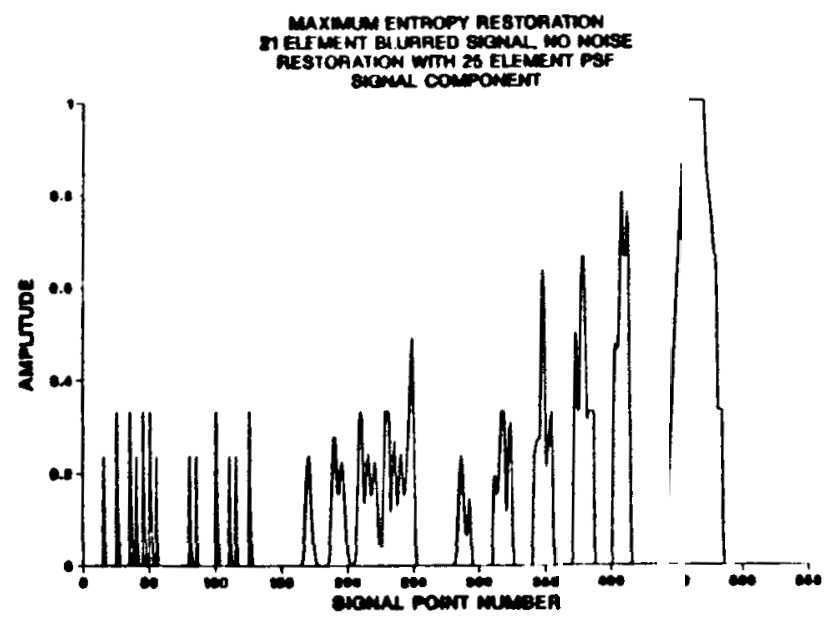

Figure 3. b) 3-segment restoration when the estimated PSF width is $19 \%$ wider than the true PSF.

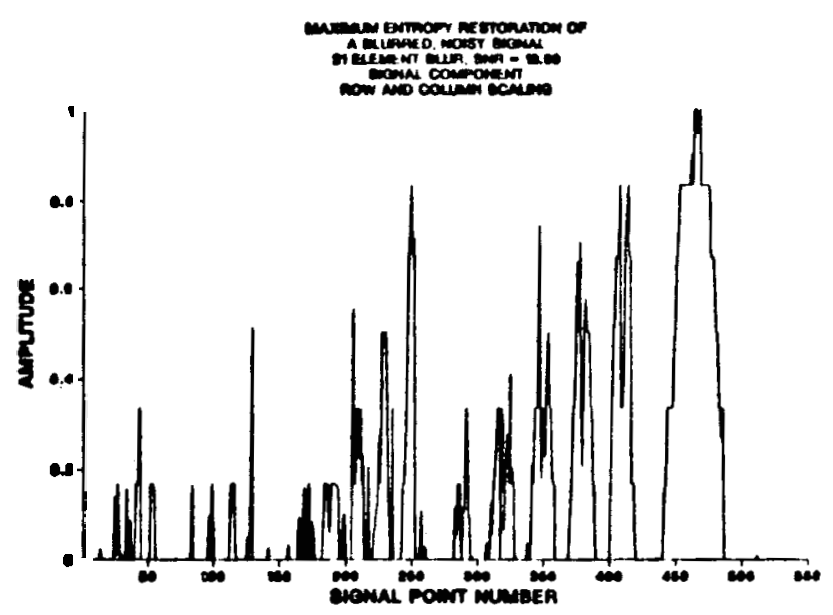

Figure 4. b) 6-segment restoration of the signal shown in Figure 4a. The true PSF is known as is the SNR.
Figure 4. a) The input blurred and noigy signal.

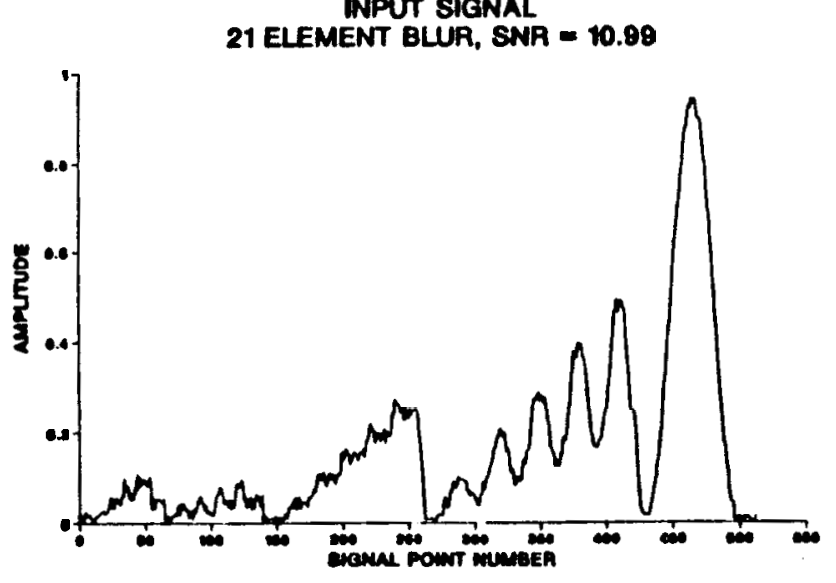




\section{Conclusion and Acknowledgments}

The LP approach using Dantzig's bounded-variable method is a viable alternative to the traditional Lagrange multiplier approach using Newton-Raphson. It's ability to employ inequality constraints may aid in achieving a basic feasible solution for very large problems. Restorations in the presence of noise degrade predictably as the SNR decreases. Likewise, errors in estimating the true PSF manifest themselves in the restored signal in an expected manner.

Special recognition must be given to Dr. Richard Hanson who designed and implemented the revised-simplex algorithm and without whose help this work could not have been done. This research was performed at Sandia National Laboratories, Albuquerque, NM, a prime contractor to the U.S. Department of Energy, under contract AC04-76DP00789.

\section{References}

1. X. Zhuang, E. Ostevold and R. M. Haralick, "The principle of maximum entropy in image recovery" in Image Recovery: Theory and Application, H. Stark, ed., Academic Press, New York, 157-193 (1987).

2. C. Kittel. Thermal Physics, Wiley and Sons, New York (1969).

3. B. R. Frieden, "Dice, entropy and likelihood," Proc. IEEE, Vol. 73, No. 12, 1764-1770 (December 1985).

4. R. Kikuchi and B. H. Soffer, "Maximum entropy image restoration. I. The entropy expression," J. Opt. Soc. Am., Vol. 67, No. 12, 1656-1665 (December 1977).

5. B. R. Frieden, "Restoring with maximum likelihood and maximum entropy," J. Opt. Soc. Am., Vol. 62, No. 4, 511-518 (April 1972).

6. S. F. Gull and G. J. Daniell, "Image reconstruction from incomplete and noisy data," Nature, Vol. 272, 686-690 (April 20, 1978).

7. S. F. Burk, S. F. Gull, and J. Skilling, "Image restoration by a powerful maximum entropy method," Comp. Vis., Graph. and Image Proc., Vol. 23, 113-128 (1983).

8. S. J. Wernecke, "Two-dimensional maximum entropy reconstruction of radio brightness," Radio Science, Vol. 12, No. 5, $831-844$ (Sept.-Oct. 1977).

9. X. Zhuang, E. Ostevold, and R. M. Haralick, “A differential equation approach to maximum entropy image reconstruction," IEEE Trans. Acous., Speech, Sig. Proc., Vol. ASSP-35, No. 2, 208-218 (Feb. 1987).

10. E. S. Meinel, "Origins of linear and nonlinear recursive restoration algorithms," J. Opt. Soc. Am. A, Vol. 3, No. 6, 787-789 (June 1986).

11. H. W. Kuhn and A. W. Tucker, "Nonlinear programming," in Proc. Second Berkeley Symp. Math. Stat. and Prob., Univ. Calif. Press, Berkeley, 481-492 (1950) and in Econometrica, Vol. 19, No. 1, 50-51 (Jan. 1951) (abstract).

12. A. W. Tucker, "Linear and nonlinear programming," Oper. Res., Vol. 5, No. 2, 244-257 (April 1957).

13. G. B. Dantrig. Linear Programming and Extensions, Princeton Univ. Press, New Jersey (1963). See Chpt. 24.

14. Available through the National Energy Software Center, Argonne National Laboratories. See also R. J. Hanson and K. L. Hiebert, "A Sparse Linear Programming Subprogram," Sandia Report SAND81-0297, Sandia National Laboratories, Albuquerque, NM (Dec. 1981). Available through NTIS, U. S. Dept. of Commerce, 5285 Port Royal Road, Springfield, VA 22161. 